\section{Dr. Yoshida, et al reply}

To the Editor:

We thank Dr. Kawada ${ }^{1}$ for his interest in our studies ${ }^{2}$. Since the landmark RRR study ${ }^{3}$, the field of biological disease-modifying antirheumatic drug (bDMARD) discontinuation study has expanded tremendously $4,5,6$. However, as Dr. Kawada pointed out, the design of studies varies widely ${ }^{6}$, making comparison of studies difficult. One important point that has to be considered in interpreting bDMARD discontinuation studies is that these studies are not necessarily examining purely biological disease processes, but rather examining the interplay of disease processes and patterns of clinical practice. Because of the rapidly changing nature of rheumatology practice in recent years, particularly in Japan, the results from our studies ${ }^{7,8}$ should be considered snapshots of a moving target.

In the study that examined the incidence of bDMARD discontinuation attempts (importantly, this study did not examine treatment success after discontinuation $)^{7}$, methotrexate (MTX) had reverse trends in the United States and Japan. That is, in the United States, physicians were more likely to attempt bDMARD discontinuation in patients who were receiving MTX, whereas the opposite was found in Japan, albeit nonsignificantly. This is likely because of the more severe underlying disease process in MTX users in Japan before the treatment guideline update that recommended more liberal use of MTX; therefore, it should not discourage physicians from discontinuing bDMARD in patients using concurrent MTX with successful treatment status.

Regarding antitumor necrosis factor (anti-TNF) bDMARD versus non-TNF bDMARD, little evidence exists on whether they differ in discontinuation practice and in persistent disease control after discontinuation, because most studies focused on the former or solely on the latter. Our studies $^{7,8}$ attempted to shed light on this by using a clinical registry including both types of users. However, the results were inconclusive in this regard because of the small sample sizes of the non-TNF bDMARD subsets. Therefore, continuing research efforts are necessary to further optimize rheumatology care.

KAZUKI YOSHIDA, MD, MPH, Department of Epidemiology, Harvard T.H. Chan School of Public Health, Boston, Massachusetts, USA; MITSUMASA KISHIMOTO, MD, PhD, Immuno-Rheumatology Center, St. Luke's International Hospital, Chuo-ku, Tokyo, Japan; DANIEL H. SOLOMON, MD, MPH, Division of Rheumatology, Immunology and Allergy, Brigham and Women's Hospital, Boston, Massachusetts, USA.
Address correspondence to Dr. K. Yoshida, Department of Epidemiology, Harvard T.H. Chan School of Public Health, 677 Huntington Ave., Boston, Massachusetts 02115, USA. E-mail: kazukiyoshida@mail.harvard.edu

\section{REFERENCES}

1. Kawada T. Predictors of biological antirheumatic drug discontinuation in patients with rheumatoid arthritis while in remission. J Rheumatol 2016;43:1253.

2. Yoshida K, Radner H, Mjaavatten MD, Greenberg JD, Kavanaugh A, Kishimoto M, et al. Incidence and predictors of biological antirheumatic drug discontinuation attempts among patients with rheumatoid arthritis in remission: A CORRONA and NinJa collaborative cohort study. J Rheumatol 2015;42:2238-46.

3. Tanaka Y, Takeuchi T, Mimori T, Saito K, Nawata M, Kameda H, et al; RRR study investigators. Discontinuation of infliximab after attaining low disease activity in patients with rheumatoid arthritis: RRR (remission induction by Remicade in RA) study. Ann Rheum Dis 2010;69:1286-91.

4. Tanaka Y, Hirata S. Intensive intervention can lead to a treatment holiday from biological DMARDs in patients with rheumatoid arthritis. Drugs 2014;74:2129-39.

5. Navarro-Millán I, Sattui SE, Curtis JR. Systematic review of tumor necrosis factor inhibitor discontinuation studies in rheumatoid arthritis. Clin Ther 2013;35:1850-61.e1.

6. Yoshida K, Sung YK, Kavanaugh A, Bae SC, Weinblatt ME, Kishimoto M, et al. Biologic discontinuation studies: a systematic review of methods. Ann Rheum Dis 2014;73:595-9.

7. Yoshida K, Radner H, Mjaavatten MD, Greenberg JD, Kavanaugh A, Kishimoto M, et al. Incidence and predictors of biological antirheumatic drug discontinuation attempts among patients with rheumatoid arthritis in remission: A CORRONA and NinJa collaborative cohort study. J Rheumatol 2015;42:2238-46.

8. Yoshida K, Kishimoto M, Radner H, Matsui K, Okada M, Saeki Y, et al. Low rates of biologic-free clinical disease activity index remission maintenance after biologic disease-modifying anti-rheumatic drug discontinuation while in remission in a Japanese multicentre rheumatoid arthritis registry. Rheumatology 2015;55:286-90.

J Rheumatol 2016;43:6; doi:10.3899/jrheum.160194 